\title{
Reduced pallidal output causes dystonia
}

\section{Atsushi Nambu ${ }^{1}$ *, Satomi Chiken ${ }^{1}$, Pullanipally Shashidharan ${ }^{2}$, Hiroki Nishibayashi $^{3}$, Mitsuhiro Ogura $^{3}$, Koji Kakishita ${ }^{3}$, Satoshi Tanaka ${ }^{3}$, Yoshihisa Tachibana ${ }^{1}$, Hitoshi Kita ${ }^{4}$ and Toru Itakura ${ }^{3}$}

Division of System Neurophysiology, National Institute for Physiological Sciences and Department of Physiological Sciences, Graduate University for Advanced Studies, Okazaki, Japan

${ }^{2}$ Department of Neurology, Mount Sinai School of Medicine, New York, NY, USA

${ }^{3}$ Department of Neurological Surgery, Wakayama Medical University, Wakayama, Japan

${ }^{4}$ Department of Anatomy and Neurobiology, College of Medicine, University of Tennessee Health Science Center, Memphis, TN, USA

\section{Edited by:}

Charles J. Wilson, University of Texas at San Antonio, USA

\section{Reviewed by:}

Thomas Wichmann, Emory University, USA

Jose Obeso, Universidad de Navarra, Spain

*Correspondence:

Atsushi Nambu, Division of System Neurophysiology, National Institute for Physiological Sciences, 38

Nishigonaka, Myodaiji, Okazaki 444-8585, Japan.

e-mail: nambu@nips.ac.jp
Dystonia is a neurological disorder characterized by sustained or repetitive involuntary muscle contractions and abnormal postures. In the present article, we will introduce our recent electrophysiological studies in hyperkinetic transgenic mice generated as a model of DYT1 dystonia and in a human cervical dystonia patient, and discuss the pathophysiology of dystonia on the basis of these electrophysiological findings. Recording of neuronal activity in the awake state of DYT1 dystonia model mice revealed reduced spontaneous activity with bursts and pauses in both internal (GPi) and external (GPe) segments of the globus pallidus. Electrical stimulation of the primary motor cortex evoked responses composed of excitation and subsequent long-lasting inhibition, the latter of which was never observed in normal mice. In addition, somatotopic arrangements were disorganized in the GPi and $\mathrm{GPe}$ of dystonia model mice. In a human cervical dystonia patient, electrical stimulation of the primary motor cortex evoked similar long-lasting inhibition in the GPi and GPe. Thus, reduced GPi output may cause increased thalamic and cortical activity, resulting in the involuntary movements observed in dystonia.

Keywords: dystonia, globus pallidus, extracellular recording, stereotactic surgery, movement disorders

\section{INTRODUCTION}

Dystonia is a neurological disorder characterized by sustained or repetitive involuntary muscle contractions and abnormal postures. The pathophysiology of dystonia is poorly understood. No consistent neuropathological or biochemical changes have been detected yet. On the other hand, abnormal neuronal activity in the basal ganglia has been reported during stereotactic surgery for deep brain stimulation (DBS) in dystonia patients (Vitek et al., 1999; Zhuang et al., 2004; Starr et al., 2005; Tang et al., 2007).

In the present article, we will introduce our recent electrophysiological studies in hyperkinetic transgenic mice generated as a model of DYT1 dystonia (Chiken et al., 2008) and in a human cervical dystonia patient (Nishibayashi et al., 2011), and discuss the pathophysiology of dystonia on the basis of these electrophysiological findings. Firstly, we investigated the neuronal activity in the entopeduncular nucleus and globus pallidus in transgenic model mice of DYT1 dystonia (Chiken et al., 2008), the most common type of human primary generalized dystonia. Secondly, we also had a chance to record neuronal activity in the internal (GPi) and external (GPe) segments of the globus pallidus of a human cervical dystonia patient during stereotactic surgery (Nishibayashi et al., 2011). The entopeduncular nucleus and globus pallidus in rodents correspond to the GPi and GPe in primates, respectively, and thus, we will call these nuclei GPi and GPe hereafter. The GPi and GPe are two important nuclei in the basal ganglia circuitry, and their abnormal neuronal activity has been reported in movement disorders. We paid special attention to the responses of GPi and GPe neurons evoked by cortical stimulation. In voluntary movements, activity originating in the cortex is transmitted through the basal ganglia circuitry and finally reaches the output station of the basal ganglia, i.e., the GPi. Cortical stimulation can mimic information processing through the basal ganglia circuitry (Nambu et al., 2002; Tachibana et al., 2008). Motor cortical stimulation typically induces triphasic responses composed of early excitation, inhibition, and late excitation in GPi and GPe neurons of normal monkeys and rodents (Yoshida et al., 1993; Nambu et al., 2000; Chiken and Tokuno, 2003). The origin of each component has been identified, with the amplitudes and durations reflecting neuronal activity of the corresponding basal ganglia pathways and nuclei. In the studies by Chiken et al. (2008) and Nishibayashi et al. (2011), long-lasting inhibition was evoked in the GPi and GPe of both DYT1 dystonia model mice and a human cervical dystonia patient.

\section{MATERIALS AND METHODS ANIMAL STUDY}

The DYT1 gene on chromosome $9 \mathrm{q} 34$ codes the torsinA protein (Ozelius et al., 1997). A three-base pair (GAG) deletion in the DYT1 gene, resulting in the loss of a glutamic acid residue $(\Delta \mathrm{E})$ in the torsinA protein (Ozelius et al., 1997), causes human DYT1 dystonia. Recently, Shashidharan et al. (2005) generated a transgenic mouse model by overexpression of human $\Delta \mathrm{E}$ torsinA. These transgenic mice developed hyperkinesia and rapid bidirectional circling. They also exhibited abnormal involuntary movements with dystonic-appearing self-clasping of limbs and head-shaking. 
In the study by Chiken et al. (2008), six DYT1 dystonia model (5-28 weeks old, both male and female) and six age-matched normal mice were used. The experimental protocols were approved by the Animal Care and Use Committees of the Mount Sinai School of Medicine and the National Institutes of Natural Sciences, and all experiments were conducted according to the guidelines of the National Institutes of Health Guide for the Care and Use of Laboratory Animals. Each mouse was anesthetized with ketamine hydrochloride $(100 \mathrm{mg} / \mathrm{kg}$ body weight, i.p.) and xylazine hydrochloride (4-5 mg/kg, i.p.), and fixed in a conventional stereotaxic apparatus. The skull was widely exposed. The exposed skull was completely covered with transparent acrylic resin, and then a small U-frame made of acetal resin for head fixation was mounted and fixed on the head of the mouse.

After recovery from the first surgery ( 2 or 3 days later), the mouse was positioned in a stereotaxic apparatus with its head restrained using the $\mathrm{U}$-frame head holder under light anesthesia with ketamine hydrochloride $(30-50 \mathrm{mg} / \mathrm{kg}$, i.p.). A part of the skull in one hemisphere was removed to access the motor cortex, GPi, and GPe. Two pairs of bipolar stimulating electrodes (tip distance, $300-400 \mu \mathrm{m}$ ) made of $50-\mu \mathrm{m}$-diameter Teflon-coated tungsten wires were inserted into the primary motor cortex, one into the caudal forelimb region and the other into the orofacial region. These regions were confirmed by observing movements evoked by intracortical microstimulation. Stimulating electrodes were then fixed therein using acrylic resin.

After full recovery from the second surgery, the mouse was positioned in a stereotaxic apparatus with its head restrained painlessly using the U-frame head holder. The mouse lay down quietly in the awake state. For single unit recording of GPi and GPe neurons, a glass-coated Elgiloy-alloy microelectrode $(0.8-1.5 \mathrm{M} \Omega$ at $1 \mathrm{kHz})$ was inserted vertically into the brain through the dura mater using a hydraulic microdrive. Signals from the electrode were amplified, converted to digital pulses using a window discriminator, and sampled using a computer. Spontaneous discharges were recorded, and spontaneous discharge rates and autocorrelograms (bin width of $0.5 \mathrm{~ms}$ ) of the neurons were calculated from continuous digitized recordings for $30 \mathrm{~s}$. Electrical stimulation of the primary motor cortex (200 $\mu$ s duration single pulse, $20-50 \mu \mathrm{A}$ strength), which induced muscle twitches in the corresponding body parts, was delivered. Similar intensities were used for dystonia model and normal mice. Responses to cortical stimulation were examined by constructing peristimulus time histograms (PSTHs; bin width of $1 \mathrm{~ms}$ ) for 100 stimulus trials.

\section{HUMAN STUDY}

In the study by Nishibayashi et al. (2011), one cervical dystonia patient received stereotactic surgery for DBS electrode implantation into the bilateral GPi. The patient was 62-year-old female, and had a disease duration of 32 months, and a Toronto western spasmodic torticollis rating scale (TWSTRS) score of 54. Microelectrode recordings were performed to identify the targets. In addition, 10 Parkinson's disease patients [eight male and two female; mean age, 61.9 years; mean disease duration, 126 months; mean levodopa dosage, $460 \mathrm{mg}$ /day; preoperative unified Parkinson's disease rating scale (UPDRS), 25.3 (best)-66.6 (worst)] were also investigated for comparison. Medications were withdrawn
$18 \mathrm{~h}$ before operation in most patients. This study was approved by the ethical committee of Wakayama Medical University and followed its guidelines.

Surgery including microelectrode recordings was performed under local anesthesia. Burr holes were made bilaterally on the coronal suture about $30 \mathrm{~mm}$ lateral from the midline. After dural incision, a strip electrode with four platinum discs (5-mmdiameter) spaced $10 \mathrm{~mm}$ apart (Unique Medical, Tokyo, Japan) was inserted into the subdural space in the posterolateral direction, and placed on the upper limb region of the primary motor cortex ipsilateral to the target GPi. Electrical stimulation (1.0 ms duration single pulse, 1-20 mA strength at $1 \mathrm{~Hz}$ ) was delivered through two of the four discs. A pair of discs inducing muscle twitches in the contralateral upper limb at the lowest intensity was selected. In the following recordings, stimulation was delivered through this pair at an intensity inducing clear muscle twitches (4-16 mA) at $1 \mathrm{~Hz}$. A microelectrode (FC1002, Medtronic, Minneapolis, MN, USA) was inserted through the same burr hole targeting the tentative target in the posteroventral GPi, which was determined on the basis of magnetic resonance imaging (MRI). Neuronal activity was amplified, displayed (Leadpoint 9033A0315, Medtronic), and fed to a computer for online analysis. The responses induced by electrical stimulation of the cortex were assessed by constructing PSTHs (bin width of $1 \mathrm{~ms}$ ) for 20-120 stimulus trials. Spontaneous discharge rates and patterns were analyzed from autocorrelograms (bin width of $0.5 \mathrm{~ms}$ ) constructed from continuous digitized recordings for $50 \mathrm{~s}$. On the basis of the microelectrode recordings, DBS electrodes (Model 3387, Medtronic) were implanted bilaterally into the GPi.

\section{RESULTS SPONTANEOUS ACTIVITY OF GPi AND GPe NEURONS IN DYT1 DYSTONIA MODEL MICE}

GPi $(50.6 \pm 15.7 \mathrm{~Hz}$, mean $\pm \mathrm{SD}, n=94$; Figure 1A) and GPe $(54.5 \pm 16.3 \mathrm{~Hz}, n=70)$ neurons in normal mice fired continuously at a high discharge rate. Traces of digitized spikes and autocorrelograms indicated that GPi (Figures 1A,B) and GPe neurons fired irregularly in normal mice. On the other hand, the firing frequency of GPi $(27.8 \pm 19.1 \mathrm{~Hz}, n=90$; Figure 1C) and GPe $(35.4 \pm 19.0 \mathrm{~Hz}, n=204)$ neurons in DYT1 dystonia model mice was significantly lower than that in normal mice (Figure 1E; $p<0.001$, Mann-Whitney U test). Discharge patterns also differed in dystonia model mice (Figures 1C,D). Bursts and pauses were frequently observed in GPi (thick black lines and thick white lines in Figure 1C) and GPe neurons of dystonia model mice.

\section{RESPONSES OF GPI AND GPE NEURONS TO CORTICAL STIMULATION IN DYT1 DYSTONIA MODEL MICE}

Cortical stimulation typically evoked a triphasic response composed of early excitation, followed by inhibition, and late excitation in GPi (Figure 2A) and GPe neurons of normal mice. On the other hand, the most common response pattern of GPi $(56 \%)$ and GPe (41\%) neurons in dystonia model mice was short-latency monophasic or biphasic excitation followed by long-lasting inhibition (Figure 2B), a pattern never observed in normal mice. The duration of the long-lasting inhibition was $73.7 \pm 29.4 \mathrm{~ms}$ in the GPi $(n=29)$ and $66.7 \pm 31.3 \mathrm{~ms}$ in the GPe $(n=46)$. 


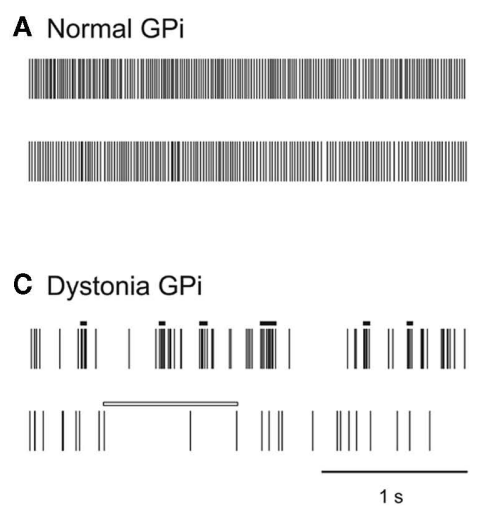

FIGURE 1 | Spontaneous activity of neurons in the internal segment of the globus pallidus (GPi) in DYT1 dystonia model mice. (A,C) Spikes are shown as digital signals in normal (A) and dystonia model (C) mice. Bursts and pauses in the digital signals are indicated by horizontal black thick lines and horizontal white thick lines, respectively. (B,D) Autocorrelograms are shown in normal (B) and dystonia model (D) mice. (E) Box plots of firing rates in normal (left) and dystonia model (right) mice. The

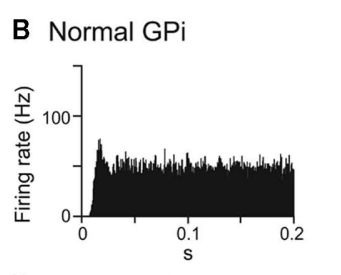

D Dystonia GPi

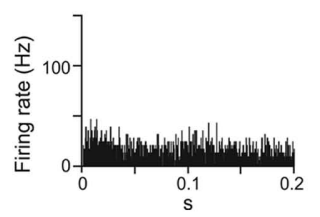

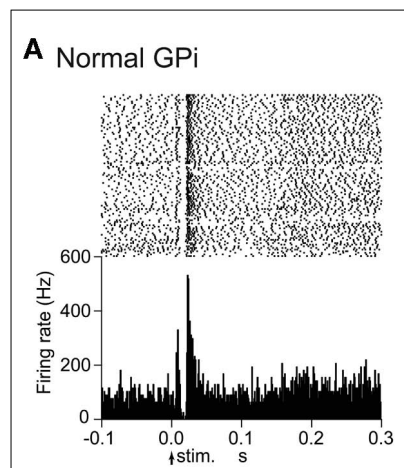

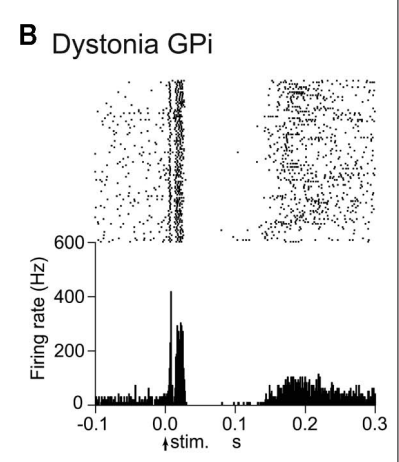

FIGURE 2 | Responses of GPi neurons to cortical stimulation in DYT1 dystonia model mice. (A) Raster and peristimulus time histograms (PSTHs) for normal mice. Cortical stimuli were delivered at time 0 (arrows) for 100 trials. (B) Raster and PSTHs for dystonia model mice. Abnormal responses with long-lasting inhibition were observed. Modified from Chiken et al. (2008).

\section{SOMATOTOPIC ORGANIZATION OF THE GPi AND GPe IN DYT1 DYSTONIA MODEL MICE}

Stimulation of both forelimb and orofacial regions of the motor cortex was performed. By observing cortically evoked responses, cortical regions projecting to each GPi neuron could be identified. In normal mice, many neurons responded to stimulation of the forelimb region, and a small number of neurons responded to stimulation of the orofacial region (Figure 3A). The number of neurons with convergent inputs from both forelimb and orofacial regions was small $(7 \%)$. On the other hand, the number of GPi neurons with convergent inputs was increased in dystonia model mice $(28 \%)$.

The locations of recorded GPi neurons are plotted on the basis of cortical inputs in Figure 3B. In the GPi of normal mice, the neurons with forelimb inputs were distributed over a wide area boxes are constructed with the top line bounding the upper quartile and the bottom line bounding the lower quartile. The median and mean excluding outliers are indicated by a thick horizontal line and an open circle in the box, respectively. The short horizontal lines show the largest and smallest values that are not outliers. Outliers are shown as small closed circles. ${ }^{*}$, significantly different $(p<0.001$, Mann-Whitney $U$ test). Modified from Chiken et al. (2008). of the GPi, although not in its most medial portion (Figure 3B top). A few neurons with orofacial inputs were found in the lateral portion of the GPi. In dystonia model mice, however, such a segregation disappeared. The number of GPi neurons with orofacial inputs and those with convergent inputs was increased, and they intruded into the central portion of the GPi, although the most medial portion remained unresponsive (Figure 3B bottom). Similar changes were also observed in the GPe. These observations suggest that somatotopic arrangements are disorganized in the GPi and GPe of dystonia model mice.

\section{ACTIVITY OF GPI AND GPe NEURONS IN A HUMAN CERVICAL DYSTONIA PATIENT}

Neurons were recorded mostly in motor territories of the GPi and GPe. The firing rates of GPi $(62.3 \pm 12.1 \mathrm{~Hz}, n=9)$ and GPe $(45.8 \pm 17.6 \mathrm{~Hz}, n=11)$ neurons in a cervical dystonia patient were significantly lower than those of GPi $(92.7 \pm 40.1 \mathrm{~Hz}$, $n=34)$ and $\mathrm{GPe}(81.0 \pm 52.5 \mathrm{~Hz}, n=17)$ neurons in Parkinsonian patients ( $p<0.05, t$-test). Most GPi and GPe neurons of a cervical dystonia patient showed burst (6/9 GPi and 5/11 GPe) or oscillatory $(1-4 \mathrm{~Hz})$ burst (1/9 GPi and 6/11 GPe) activity (Figure 4A). More than one-third of recorded neurons (6/13 GPi and 4/11 GPe in a cervical dystonia patient and 21/68 GPi and 18/45 GPe in Parkinsonian patients) showed responses to cortical stimulation. These GPi and GPe neurons were considered to be located in the upper limb regions of the GPi and GPe, because these neurons often responded to sensory stimulation of the upper limb. In Parkinsonian patients, response patterns to cortical stimulation were combinations of early excitation, inhibition, and late excitation (data not shown). On the other hand, in a cervical dystonia patient, long-lasting inhibition preceded by excitation (Figure 4B) and long-lasting monophasic inhibition (Figure 4C) were the typical response patterns. These response patterns are very similar to those observed in dystonia model mice (compare Figures 4B,C with Figure 2B). 

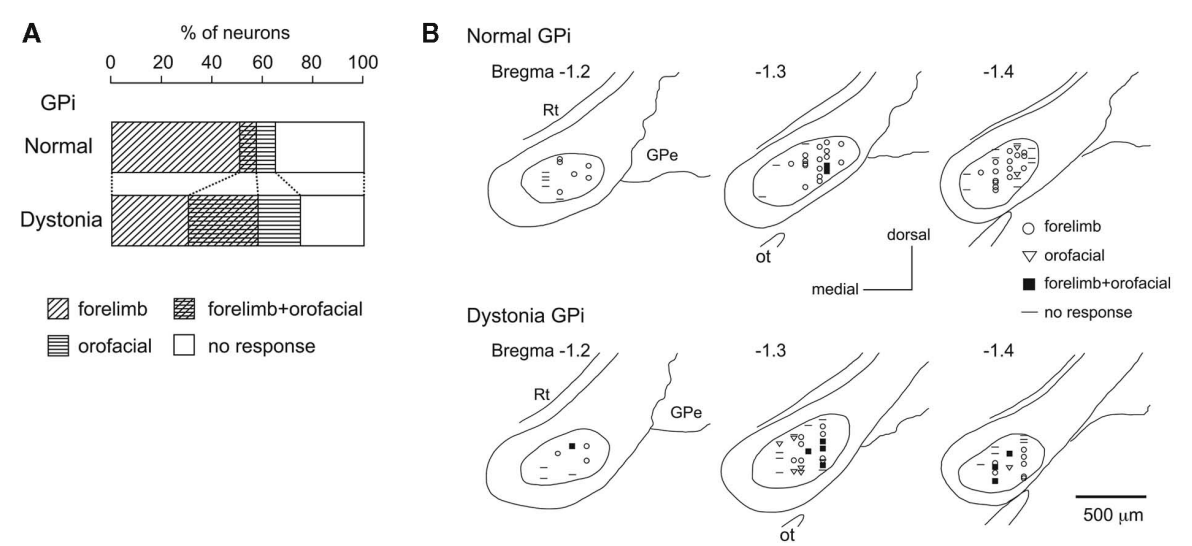

FIGURE 3 | Somatotopic organization in the GPi of DYT1 dystonia model mice. (A) Proportions of neurons classified on the basis of cortical inputs in normal (top) and dystonia model (bottom) mice. (B) Distribution of recorded GPi neurons indicated by symbols on the basis of cortical inputs. Data from normal (top) and dystonia model (bottom) mice are shown in frontal sections. Figures in the left upper corner represent distance from bregma. GPe, external segment of the globus pallidus; Rt, reticular thalamic nucleus; Ot, optic tract. Modified from Chiken et al. (2008).
FIGURE 4 | Activity of GPi and GPe neurons in a human cervical dystonia patient. (A) Autocorrelograms and slow traces of digitized spikes of GPe neurons recorded from a cervical dystonia (CD) patient. (B,C) PSTHs (bin width of $1 \mathrm{~ms}$ ) showing the responses of GPi (B) and $\mathrm{GPe}$ (C) neurons evoked by stimulation of the upper limb region of the primary motor cortex in a cervical dystonia patient. Cortical stimuli

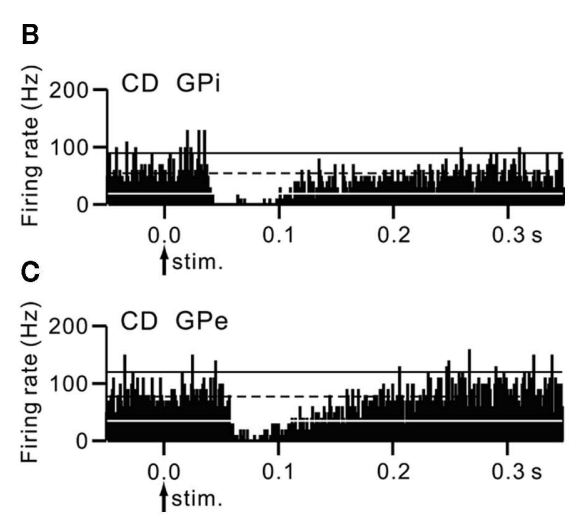

were delivered at time 0 (arrows) for 100 trials. The mean firing rate is indicated by black dotted lines. The statistical levels of $p<0.05$ (one-tailed $t$-test) calculated from the firing rate during $100 \mathrm{~ms}$ preceding the onset of stimulation are indicated by black and white solid lines (upper and lower limits of $p<0.05$ ). Modified from Nishibayashi et al. (2011).

\section{DISCUSSION}

The first part of the present article characterized the electrophysiological properties of transgenic mice developed to express human $\triangle \mathrm{E}$-torsinA as a model of DYT1 dystonia. These mice exhibited: (1) decreased GPi and GPe activity with bursts and pauses, (2) cortically evoked long-lasting inhibition in the GPi and GPe, and (3) somatotopic disorganization in the GPi and GPe. In the second part, similar activity changes, such as decreased activity with bursts and cortically evoked long-lasting inhibition, were also observed in the GPi and GPe of a human cervical dystonia patient. These neuronal abnormalities may be responsible for the symptoms observed in dystonia.

\section{DECREASED GPi AND GPe ACTIVITY IN DYSTONIA}

In the present article, reduction of the spontaneous firing rates of GPi and GPe neurons was observed in dystonia model mice and a human cervical dystonia patient. Alteration of firing patterns was also observed in both of them, including bursting discharges and pauses. Decreased discharge rates and irregularly grouped discharges with intermittent pauses in GPi and GPe neurons were also reported in patients with generalized dystonia (Vitek et al., 1999; Zhuang et al., 2004; Starr et al., 2005) and cervical dystonia (Tang et al., 2007). Dystonic hamsters with paroxysmal generalized dystonia also exhibited reduced and bursting GPi activity (Gernert et al., 2002). The correlation between abnormal neuronal activity and abnormal movements was not investigated in the present mice study, because it was difficult to observe abnormal movements under head fixation. The mechanisms responsible for decreased firing rates may include: (1) alteration of membrane properties of GPi and GPe neurons, (2) increased inhibitory inputs to the GPi and GPe, such as GABAergic inputs from the striatum, and/or (3) decreased excitatory inputs to the GPi and 
GPe, such as glutamatergic inputs from the subthalamic nucleus (STN). Inhibitory inputs from the striatum to the GPi and GPe were increased in dystonia model mice as discussed in the next section.

\section{CORTICALLY EVOKED LONG-LASTING INHIBITION IN GPi AND GPe NEURONS OF DYSTONIA}

In normal mice, cortical stimulation typically induced triphasic responses composed of early excitation, inhibition, and late excitation in GPi and GPe neurons. Similar triphasic responses were also observed in the GPi and GPe of rats and monkeys. The origin of each component has been intensively studied (Ryan and Clark, 1991; Maurice et al., 1998, 1999; Nambu et al., 2000; Kita et al., 2004; Tachibana et al., 2008). Early excitation is mediated by the cortico-STN-GPe/GPi pathway, while inhibition and late excitation are mediated by the cortico-striato-GPe/GPi and cortico-striato-GPe-STN-GPe/GPi pathways, respectively.

On the other hand, in dystonia model mice we examined, cortical stimulation induced early excitation followed by late longlasting inhibition in GPi and GPe neurons. Similar response patterns were induced in GPi and GPe neurons of a human cervical dystonia patient. These abnormal patterns of responses may be generated through the cortico-basal ganglia pathways. The early excitation seems to be mediated, at least in its early phase, by the cortico-STN-GPe/GPi pathway, as in normal mice, since the latency of the early excitation in dystonia model mice was short and similar to that in normal mice. The origin of the late longlasting inhibition may be (1) increased inhibitory input via the striato-GPe/GPi pathway, or (2) decreased excitatory input via the STN-GPe/GPi pathway. The latter explanation seems less likely to be correct, since our preliminary observation indicates that the spontaneous activity of STN neurons is unchanged in dystonia model mice. Thus, increased activity through both cortico-striatoGPi direct and cortico-striato-GPe indirect pathways is considered to be the fundamental change in dystonia. The above observations also suggest that spontaneous excitation in the cortex that is transmitted to the GPi and GPe through the cortico-basal ganglia pathways could also induce short-latency excitation and longlasting inhibition, which might be the origins of bursts and pauses, respectively.

\section{SOMATOTOPIC DISORGANIZATION IN THE GPi AND GPe OF DYSTONIA}

The somatotopic organization in the GPi and GPe was observed in normal mice as well as monkeys (DeLong et al., 1985; Yoshida et al., 1993). On the other hand, in dystonia model mice, somatotopic arrangements were disorganized, and many GPi and GPe neurons received convergent inputs from both forelimb and orofacial regions. Widened somatosensory receptive fields in pallidal neurons were reported in patients with generalized (Vitek et al., 1999) and focal (Lenz et al., 1998; Sanger et al., 2001) dystonia. Interference of information processing may occur through the cortico-basal ganglia pathways. One explanation for this could be that each single GPi or GPe neuron receives inputs from more striatal neurons in dystonia model mice than in normal mice. Such an explanation agrees well with the hypothesis that in dystonia, inhibition in the GPi/GPe is increased through the striato-GPe/GPi pathways as described in the previous section.

\section{PATHOPHYSIOLOGY OF DYSTONIA}

The GPi, the output nucleus of the basal ganglia, is composed of GABAergic inhibitory neurons and fires at high frequency in normal states. Its target structures, such as the thalamus and frontal cortex, are thus continuously inhibited. Striatal inputs reduce GPi activity in a temporal fashion, excite thalamic and cortical neurons through disinhibitory mechanism, and finally release appropriate movements with appropriate timing (Figure 5A; Nambu et al., $2000,2002)$. On the other hand, in dystonia model mice and a human cervical dystonia patient, cortical excitation induced longlasting inhibition in the GPi (Figure 5B). This suggests that even tiny amounts of spontaneous or voluntary neuronal activity originating in the cortex are transmitted through the cortico-basal ganglia pathways to induce strong, long-lasting inhibition in the GPi. Moreover, somatotopic disorganization was noted in the GPi of dystonia, and cortical activation could induce inhibition over a wide area of the GPi. Reduced GPi output may activate wide areas of the thalamus and cortex in an uncontrollable fashion, resulting in the involuntary movements observed in dystonia. In a similar manner, the cortical areas controlling agonist and antagonist muscles are concurrently activated, and co-contraction of agonist and antagonist muscles could be induced. This may also explain the "motor overflow" that is unintentional muscle contraction during voluntary movements in dystonia. Activation of the upper limb region in the motor cortex, for example, may inhibit large areas of the GPi and finally induce involuntary movements of multiple body parts (Figure 5B).

\section{CONCLUSION}

The activity of GPi and GPe neurons in DYT1 dystonia model mice and a human cervical dystonia patient was investigated. Both of them showed similar activity changes, such as decreased spontaneous activity with bursts and long-lasting inhibition evoked by cortical stimulation, indicating increased activity through
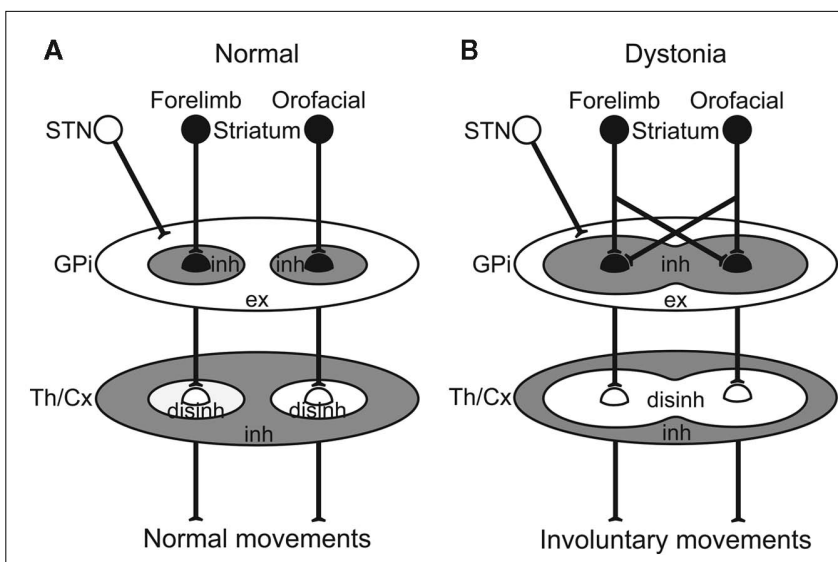

FIGURE 5 | Schematic diagrams showing information processing through the basal ganglia in normal $(A)$ and dystonia (B) conditions. In dystonia (B), cortical activation induces strong inhibition over a wide area of the GPi. Reduced GPi output may cause strong excitation in the thalamus (Th) and cortex $(\mathrm{Cx})$ through disinhibitory mechanism, resulting in the involuntary movements. disinh, disinhibition; ex, excitation; inh, inhibition; STN, subthalamic nucleus. Modified from Chiken et al. (2008). 
the cortico-striato-GPi direct and cortico-striato-GPe indirect pathways. Such a mechanism may explain the pathophysiology of dystonia: Neuronal activity originating in the cortex is transmitted through the cortico-basal ganglia pathways to induce strong, long-lasting inhibition in the GPi. Reduced GPi output may cause increased thalamic and cortical activity, resulting in the involuntary movements.

\section{ACKNOWLEDGMENTS}

The animal study was supported by a Grants-in-Aid for Exploratory Research (18650089) and a Grants-in-Aid for Scientific Research (B) (18300135) from the Ministry of Education,

\section{REFERENCES}

Chiken, S., Shashidharan, P., and Nambu, A. (2008). Cortically evoked long-lasting inhibition of pallidal neurons in a transgenic mouse model of dystonia. J. Neurosci. 28, 13967-13977.

Chiken, S., and Tokuno, H. (2003). Ablation of striatal interneurons influences activities of entopeduncular neurons. Neuroreport 14, 675-678.

DeLong, M. R., Crutcher, M. D., and Georgopoulos, A. P. (1985). Primate globus pallidus and subthalamic nucleus: functional organization. J. Neurophysiol. 53, 530-543.

Gernert, M., Bennay, M., Fedrowitz, M., Rehders, J. H., and Richter, A. (2002). Altered discharge pattern of basal ganglia output neurons in an animal model of idiopathic dystonia. J. Neurosci. 22, 7244-7253.

Kita, H., Nambu, A., Kaneda, K., Tachibana, Y., and Takada, M. (2004). Role of ionotropic glutamatergic and GABAergic inputs on the firing activity of neurons in the external pallidum in awake monkeys. J. Neurophysiol. 92, 3069-3084.

Lenz, F. A., Suarez, J. I., Metman, L. V., Reich, S. G., Karp, B. I., Hallett, M., Rowland, L. H., and Dougherty, P. M. (1998). Pallidal activity during dystonia: somatosensory reorganisation and changes with severity. J. Neurol. Neurosurg. Psychiatr. 65, 767-770.

Maurice, N., Deniau, J. M., Glowinski, J., and Thierry, A. M. (1998). Relationships between the prefrontal cortex and the basal ganglia in the rat: physiology of the corticosubthalamic circuits. J. Neurosci. 18, 9539-9546.

Maurice, N., Deniau, J. M., Glowinski, J., ships between the prefrontal cortex and the basal ganglia in the rat: physiology of the cortico-nigral circuits. J. Neurosci. 19, 4674-4681.

Nambu, A., Tokuno, H., Hamada, I., Kita, H., Imanishi, M., Akazawa, T., Ikeuchi, Y., and Hasegawa, N. (2000). Excitatory cortical inputs to pallidal neurons via the subthalamic nucleus in the monkey. J. Neurophysiol. 84, 289-300.

Nambu, A., Tokuno, H., and Takada, M. (2002). Functional significance of the cortico-subthalamo-pallidal 'hyperdirect' pathway. Neurosci. Res. 43, 111-117.

Nishibayashi, H., Ogura, M., Kakishita, K., Tanaka, S., Tachibana, Y., Nambu, A., Kita, H., and Itakura, T. (2011). Cortically evoked responses of human pallidal neurons recorded during stereotactic neurosurgery. Mov. Disord. 26, 469-476.

Ozelius, L. J., Hewett, J. W., Page, C. E., Bressman, S. B., Kramer, P. L., Shalish, C., de Leon, D., Brin, M. F., Raymond, D., Corey, D. P., Fahn, S., Risch, N. J., Buckler, A. J., Gusella, J. F., and Breakefield, X. O. (1997). The early-onset torsion dystonia gene (DYT1) encodes an ATP-binding protein. Nat. Genet. 17, 40-48.

Ryan, L. J., and Clark, K. B. (1991). The role of the subthalamic nucleus in the response of globus pallidus neurons to stimulation of the prelimbic and Thierry, A. M. (1999). Relation-

Culture, Sports, Science, and Technology of Japan (MEXT), The Uehara Memorial Foundation, Takeda Science Foundation, and United States-Japan Brain Research Cooperative Program (Atsushi Nambu); a Grants-in-Aid for Scientific Research (C) (19500354) from MEXT (Satomi Chiken); and National Institute of Neurological Disorders and Stroke-National Institutes of Health Grant (NS-043038) (Pullanipally Shashidharan). The human study was supported by Wakayama Foundation for the Promotion of Medicine (Toru Itakura), a Grants-in-Aid for Scientific Research (B) (18300135) from MEXT and the Uehara Memorial Foundation (Atsushi Nambu), and NIH grants (NS-47085 and NS-57236) (Hitoshi Kita).

and agranular frontal cortices in rats. Exp. Brain Res. 86, 641-651.

Sanger, T. D., Tarsy, D., and PascualLeone, A. (2001). Abnormalities of spatial and temporal sensory discrimination in writer's cramp. Mov. Disord. 16, 94-99.

Shashidharan, P., Sandu, D., Potla, U., Armata, I. A., Walker, R. H., McNaught, K. S., Weisz, D., Sreenath, T., Brin, M. F., and Olanow, C. W. (2005). Transgenic mouse model of early-onset DYT1 dystonia. Hum. Mol. Genet. 14, 125-133.

Starr, P. A., Rau, G. M., Davis, V., Marks, W. J. Jr., Ostrem, J. L., Simmons, D., Lindsey, N., and Turner, R. S. (2005). Spontaneous pallidal neuronal activity in human dystonia: comparison with Parkinson's disease and normal macaque. J. Neurophysiol. 93, 3165-3176.

Tachibana, Y., Kita, H., Chiken, S., Takada, M., and Nambu, A. (2008). Motor cortical control of internal pallidal activity through glutamatergic and GABAergic inputs in awake monkeys. Eur. J. Neurosci. 27, 238-253.

Tang, J. K., Moro, E., Mahant, N., Hutchison, W. D., Lang, A. E., Lozano, A. M., and Dostrovsky, J. O. (2007). Neuronal firing rates and patterns in the globus pallidus internus of patients with cervical dystonia differ from those with Parkinson's disease. J. Neurophysiol. 98, 720-729.

Vitek, J. L., Chockkan, V., Zhang, J. Y., Kaneoke, Y., Evatt, M., DeLong, M. R., Triche, S., Mewes, K., Hashimoto, T., and Bakay, R. A. E. (1999). Neuronal activity in the basal ganglia in patients with generalized dystonia and hemiballismus. Ann. Neurol. 46, 22-35.

Yoshida, S., Nambu, A., and Jinnai, K. (1993). The distribution of the globus pallidus neurons with input from various cortical areas in the monkeys. Brain Res. 611, 170-174.

Zhuang, P., Li, Y., and Hallett, M. (2004). Neuronal activity in the basal ganglia and thalamus in patients with dystonia. Clin. Neurophysiol. 115, 2542-2557.

Conflict of Interest Statement: The authors declare that the research was conducted in the absence of any commercial or financial relationships that could be construed as a potential conflict of interest.

Received: 21 April 2011; accepted: 18 October 2011; published online: 28 November 2011.

Citation: Nambu A, Chiken S, Shashidharan $P$, Nishibayashi $H$, Ogura $M$, Kakishita K, Tanaka S, Tachibana $Y$, Kita $H$ and Itakura $T$ (2011) Reduced pallidal output causes dystonia. Front. Syst. Neurosci. 5:89. doi: 10.3389/fnsys.2011.00089

Copyright (c) 2011 Nambu, Chiken, Shashidharan, Nishibayashi, Ogura, Kakishita, Tanaka, Tachibana, Kita and Itakura. This is an open-access article subject to a non-exclusive license between the authors and Frontiers Media SA, which permits use, distribution and reproduction in other forums, provided the original authors and source are credited and other Frontiers conditions are complied with. 